\title{
O ENSINO DE BIM NO BRASIL: CENÁRIO DOS ESTUDOS DE PÓS- GRADUAÇÃO LATO SENSU
}

\author{
Elaine Estevam Teleginski-elaine.teleginski@gmail.com \\ UTFPR - Universidade Tecnológica Federal do Paraná \\ Curitiba-PR
}

Fábio Freire-fabiofreire@utfpr.edu.br

UTFPR - Universidade Tecnológica Federal do Paraná

Curitiba-PR

\section{Resumo}

$\mathrm{Na}$ atualidade, muitas empresas e profissionais da indústria AECO (Arquitetura, Engenharia, Construção e Operação) tem utilizado o BIM (Modelagem da Informação da Construção) no desenvolvimento de projetos, mas que em muitos casos, acaba por produzir informações desencontradas acerca de seus conceitos. Neste sentido, o presente estudo procura identificar a oferta de cursos de pós-graduação lato sensu em BIM, no contexto brasileiro. Os cursos em nível de especialização no Brasil são denominados de pós-graduação lato sensu, em oposição aos cursos stricto sensu, que é o modo utilizado para denominar os cursos em nível de mestrado e doutorado. Os cursos de especialização são essenciais para o aprimoramento do conhecimento dos profissionais que já atuam no mercado ou que querem se aprofundar em áreas mais especificas. Com base em pesquisa realizada na internet foram obtidos dados a respeito da oferta de cursos de especialização com predominância de conteúdo BIM, aqui considerados como aqueles que possuem, no mínimo, $50 \%$ de sua carga horária/disciplinas adotando BIM em suas matrizes curriculares. Além dos dados obtidos acerca da carga horária, corpo docente, disciplinas e local dos cursos, foi investigado a adoção do conceito OpenBIM como método de ensino-aprendizagem. São apresentados alguns dados e os principais obstáculos para o ensino de BIM voltado a capacitação, em nível de especialização, de profisssionais da AECO no Brasil.

Palavras-chave: BIM. Pós-graduação em BIM. Especialista BIM.

\section{BIM TEACHING IN BRAZIL: SCENARIO OF LATO SENSU POSTGRADUATE STUDIES}

\begin{abstract}
Currently, many companies and professionals in the AECO industry (Architecture, Engineering, Construction and Operation) have used BIM (Construction Information Modeling) in project development, but in many cases, it ends up producing mismatched information about their concepts. . In this sense, the present study seeks to identify the offer of lato sensu postgraduate courses in BIM, in the Brazilian context. Specialization-level courses in Brazil are called lato sensu postgraduate courses, as opposed to stricto sensu courses, which is the way used to name courses at the master's and doctoral level. Specialization courses are essential for improving the knowledge of professionals who already work in the market or who want to deepen in more specific areas. Based on research conducted on the Internet, data were
\end{abstract}


obtained regarding the offer of specialization courses with a predominance of BIM content, considered here as those that have at least $50 \%$ of their workload / disciplines adopting BIM in their curricular matrices. In addition to the data obtained about the workload, faculty, disciplines and location of the courses, the adoption of the OpenBIM concept as a teaching-learning method was investigated. Some data are presented and the main obstacles to the teaching of BIM aimed at training, at the level of specialization, of AECO professionals in Brazil.

Keywords: BIM. Postgraduate studies in BIM. BIM specialist.

\section{INTRODUÇÃO}

A crescente disseminação do BIM (Modelagem da Informação da Construção) no Brasil, evidencia a importância de qualificação dos profissionais da AECO (Arquitetura, Engenharia, Construção e Operação) para atender as necessidades de modernização da indústria da construção civil. No entanto, mesmo com o aumento do uso da tecnologia, a utilização do BIM ainda é isolada e focada em softwares ao invés do processo (JAYME; FREIRE, 2019). Succar (2009) define três estágios na adoção do BIM: o primeiro, onde nos encontramos atualmente, o foco está na modelagem. O segundo, denominado estágio 2 (BIM 2.0) refere-se à colaboração, e finalmente, nível 3 (BIM 3.0) definido pela prática integrada dos processos e colaboradores. Diante deste cenário é inegável a necessidade de oferta de cursos voltados a capacitação profissional, em ferramentas digitais, para obter todo o potencial do processo BIM.

O BIM ainda não vem sendo inserido, de forma consistente, nos currículos dos cursos de graduação em Arquitetura e Engenharia Civil nas universidades brasileiras (BARISON, 2015). Nessa mesma linha, segundo Jayme e Freire (2019) apenas 10 (dez) de 53 (cinquenta e três) cursos de graduação em Arquitetura, em instituições públicas no Brasil, apresentam disciplinas obrigatórias que envolvem conteúdos BIM e outros 05 (cinco) cursos incluem apenas disciplinas optativas, grupos de estudos ou laboratórios especializados.

Diante deste contexto, a demanda por cursos de especialização em BIM tem ganhado atenção das instituições de ensino superior, que em muitos casos não possuem corpo docente qualificado, mas que vislumbram uma excelente oportunidade de ganhos financeiros.

A Resolução CES/CNE no 1 de 2018 do Ministério da Educação estabelece diretrizes e normas para a oferta dos cursos de pós-graduação lato sensu, denominados cursos de especialização no Brasil. Define-se como cursos de especialização os programas de nível superior, de educação continuada, com objetivos de complementar a formação acadêmica, atualizar, incorporar competências técnicas e desenvolver novos perfis profissionais, com vistas ao aprimoramento da atuação no mundo do trabalho e ao atendimento de demandas por profissionais tecnicamente mais qualificados para o setor público, empresas e organizações do terceiro setor, tendo em vista o desenvolvimento do país.

Importante destacar que, a Estratégia Nacional de Disseminação do Building Information Modelling no Brasil - Estratégia BIM BR, instituída pelo Governo Federal em 2018, definiu como parte dos seus objetivos estimular a capacitação em BIM, fomentando a inserção do BIM nas disciplinas dos cursos de graduação e pós-graduação em Arquitetura e Engenharias, dado que, "a utilização do BIM exige que o profissional tenha conhecimento desse novo processo e esteja capacitado para as implicações decorrentes dessa mudança de paradigma. Isso é fator fundamental para que o BIM seja efetivamente compreendido, adotado e consolidado no mercado brasileiro" (BRASIL, 2018b).

\section{OBJETIVOS E METODOLOGIA}

Este estudo propõe identificar os cursos de especialização com predominância de conteúdo 
BIM (com o mínimo de $50 \%$ de sua carga horária destinada ao tema) ofertados no Brasil, com o objetivo de entender como os cursos existentes se propõe a capacitar os profissionais da AECO para o mercado de trabalho, cada vez mais exigente e complexo.

O método utilizado para levantamento dos dados foi o de consulta as páginas eletrônicas, dos cursos de especialização que mencionam o tema BIM, encontrados em buscas realizadas na internet. Não foram considerados os cursos de especialização que continham menos de $50 \%$ da totalidade de disciplinas/carga horária envolvendo o tema BIM.

Importante destacar que os dados foram obtidos a partir das informações disponíveis nas páginas eletrônicas dos cursos de especialização, e que, em alguns casos eram insuficientes.

\section{RESULTADOS}

A partir do levantamento realizado foram identificados 27 (vinte e sete) cursos de pósgraduação em BIM, ofertados por 26 (vinte e seis) instituições de ensino superior (IES) públicas (12\%) e privadas (88\%), são elas: PUC Minas, UNINTER, Inbec, Unyleya, IPOG, SENAI CIMATEC, IBEC, Zigurat/UNIFEBE, UNIP, TOTH/ESB, UTFPR, Anhembi Morumbi, Instituto Mauá, UNASP, UNIFACS, ISITEC, IDD, UNB/PECC, RTG, UNIFOR, Monte Pascoal/INCURSOS, Faculdade NOVOESTE, SENAI-PB, Universidade Potiguar, FACENS e USCS. O objetivo da maioria dos cursos é no sentido da capacitação de profissionais, utilizando softwares, para modelagem, documentação, compatibilização e planejamento, normalmente por meio do desenvolvimento de modelos 3D, capacitando-os principalmente como modeladores BIM.

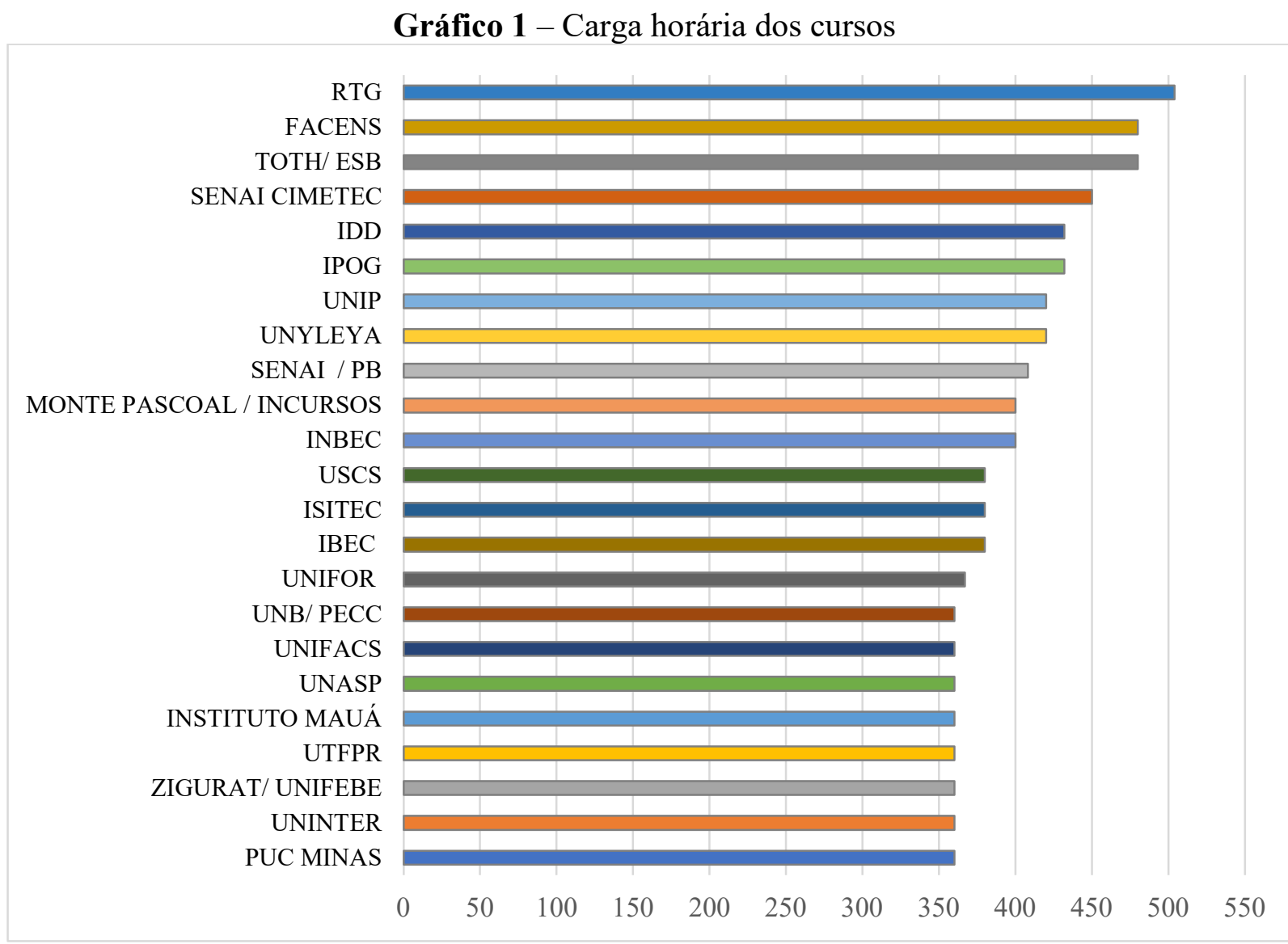

Fonte - Os Autores 
Conforme definido na Resolução CES/CNE $n^{0} 1$, a matriz curricular deve ter a carga mínima de 360 (trezentos e sessenta) horas (BRASIL, 2018a). No entanto, a grande maioria dos cursos possuem carga horária superior a $360 \mathrm{~h}$, chegando em alguns casos a ultrapassar $500 \mathrm{~h}$ (Ver Gráfico 1). Foi possível verificar que os cursos com maior carga horária incluem como disciplina o Trabalho de Conclusão de Curso (TCC). Das 26 (vinte e seis) IES, objeto do estudo, 03 (três) não informaram nas suas páginas eletrônicas o carga horaria do curso, são elas: Universidade Potiguar, Anhembi Morumbi e Faculdade NOVOESTE.

Gráfico 2 - Titulação do corpo docente

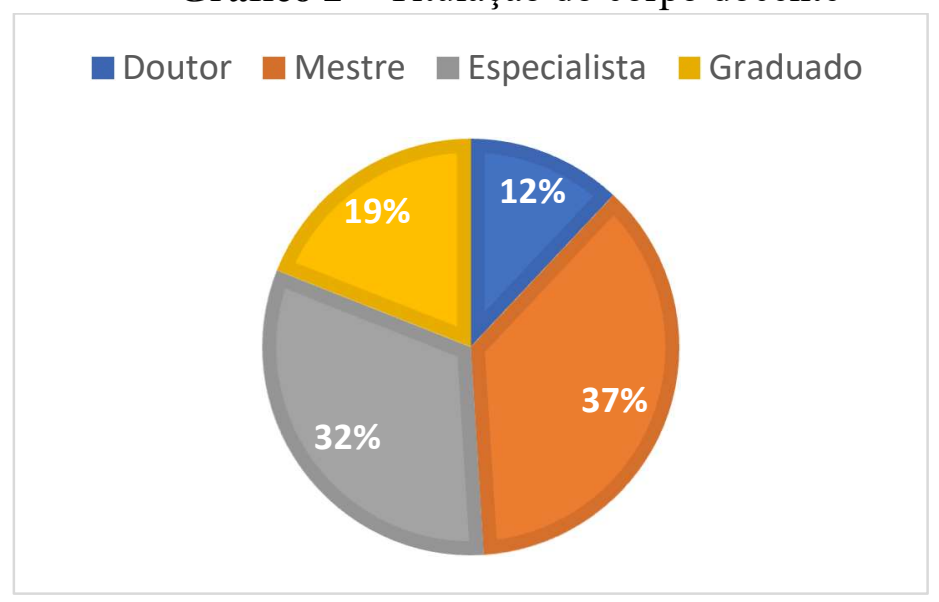

Fonte - Os Autores

A Resolução CES/CNE $n^{\circ} 1$ (BRASIL, 2018a) estabelece que o corpo docente do curso de especialização deve ser constituído por, no mínimo, 30\% (trinta por cento) de portadores do título de pós-graduação stricto sensu obtidos em instituições devidamente reconhecidas pelo poder público. Esta exigência não significa conhecimento técnico, mas por ser um critério do MEC, deve ser cumprido por todas as IES. Grande parte dos professores que compõem o corpo docente dos cursos, cuja informação estava acessível nas páginas eletrônicas, é formada por mestres $(37 \%)$ e doutores $(12 \%)$. Também foi possível identificar que há enorme contigente de professores com apenas graduação e/ou especialização (Ver Grafico 2), o que pressupõe na maioria das vezes, atuação profissional em empresas da construção civil.

Gráfico 3 - Perfil das IES

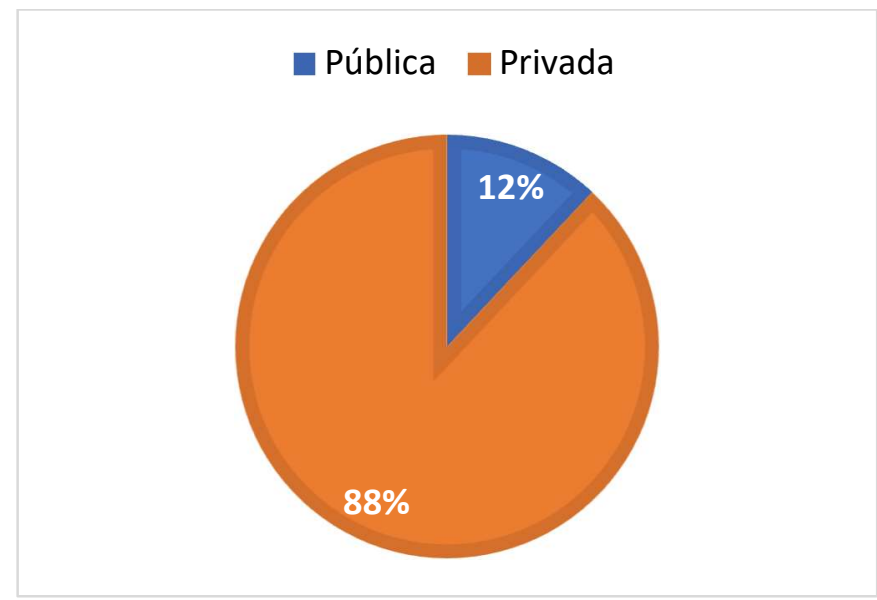

Fonte - Os Autores 
A maior parte dos cursos disponíveis no Brasil são ofertados por instituições privadas, totalizando 23 (vinte e três) das 26 (vinte e seis) identificadas no estudo (Ver Gráfico 3). As principais dificuldades na oferta de cursos em instituições públicas, que representa algo em torno de $12 \%$ do total, consiste na necessidade de infraestrutura adequada, dificuldade de colaboração entre os cursos de arquitetura e engenharias, e finalmente, carência de professores com capacitação e interesse em adotar o processo BIM (BENEDETTO; BERNARDES; PIRES, 2016).

Gráfico 4 - Oferta de cursos por regiões

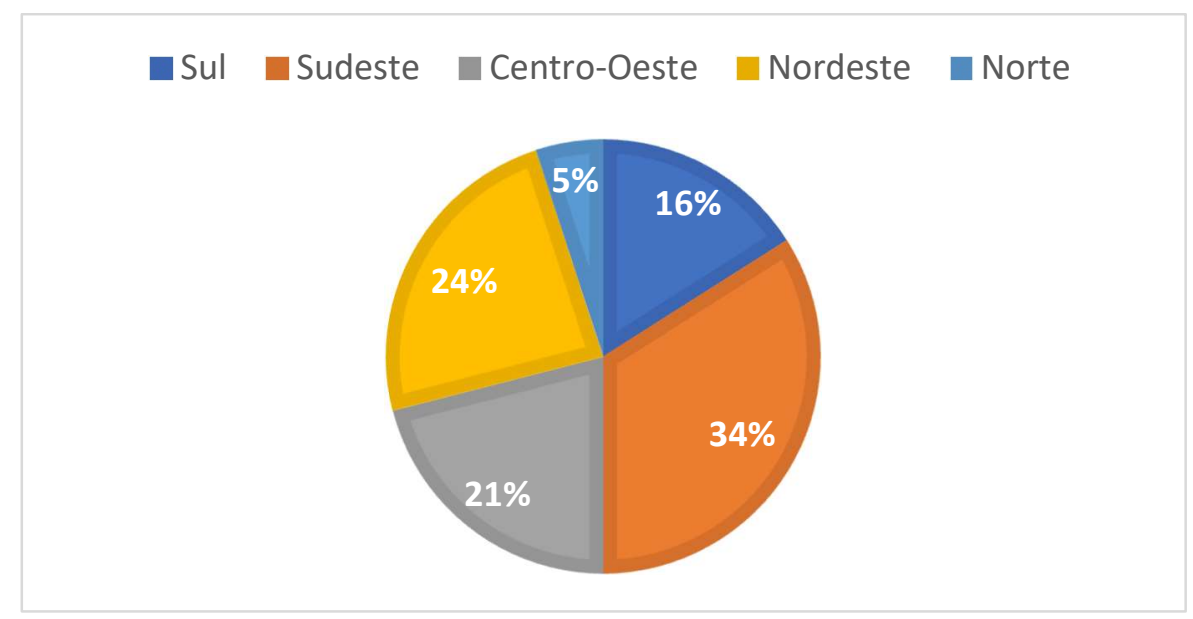

Fonte - Os Autores

Algumas IES oferecem cursos presenciais em mais de uma localidade, enquanto outras possuem apenas a modalidade EAD (ensino à distância). Dos cursos presenciais identificados, a maioria concentra-se na região Sudeste, seguido da região Nordeste, Centro-Oeste, Sul e Norte, com apenas 5\% (Ver Gráfico 4).

A grade de disciplinas, dos cursos ofertados, segue um padrão comum e assemelha-se as etapas do processo construtivo real, iniciando com a disciplina de projeto arquitetônico, seguido por disciplinas que abordam os projetos de estruturas e instalações prediais, compatibilização, orçamentação e planejamento, eventualmente outros temas são abordados. Segundo Ruschel (2018), a pós-graduação visa formar especialistas em Modelagem da Informação da Construção com competências para produção e interpretação de modelos, processo colaborativo, estimativa de custos, sequenciamento de atividades de construção, análises, coordenação 3D e a gestão de facilidades. Tembém adquirindo conhecimento em relação as estratégias para implementação de BIM e diretrizes para desenvolvimento e contratação de modelos para os diversos usos experimentados.

Verificando com mais atenção a grade curricular dos cursos, a maioria possui disciplinas que apresentam as principais aplicações do BIM na indústria AECO, tecnologias inovadoras e procedimentos para implantação BIM em empresas. Foi possível, também, encontrar cursos que oferecem disciplinas sobre conteúdos específicos, tais como: urbanismo, arquitetura de interiores, patrimônio cultural, luminotécnica e infraestrutura.

Segundo Addor (2010), o processo BIM pressupõe envolvimento e comunicação de vários agentes ao longo de todo o ciclo de vida da edificação. Levando em consideração que BIM não é software, é indispensável que os cursos tenham compromisso com padrões abertos para troca 
de informações entre as diversas plataformas, evitando deste modo, que empresas e profissionais da indústria AECO fiquem reféns de formatos nativos. Diante do exposto, se faz necessário que os cursos tenham iniciativas para adoção do OpenBIM (E-ZIGURAT, 2019).

Apesar da relevância do conceito OpenBIM, apenas a UTFPR, dos 27 (vinte e sete) cursos identificados, destaca o fluxo de trabalho OpenBIM na apresentação do seu programa de pósgraduação lato sensu.

Os softwares BIM de maior preferência, indicados nos cursos pesquisados neste estudo, são os seguintes: Revit, ARCHICAD, Solibri e Tekla Structures. Vale ressaltar, que o curso ofertado pela UTFPR apresenta a maior diversidade de softwares utilizados, provavelmente em razão da ênfase no fluxo de trabalho OpenBIM.

\section{CONSIDERAÇÕES FINAIS}

De acordo com o levantamento de dados obtido pela consulta às páginas eletrônicas dos cursos de pós-graduação lato sensu, que tem relação com o processo BIM, ofertados no Brasil, ainda é parcial. Isto se deve a ausência de inúmeras informações nas páginas eletrônicas dos cursos. Apesar disso, o ensino de BIM, em nível de especialização no Brasil, apresenta uma perspectiva que ainda patrocina a utilização de softwares BIM da empresa Autodesk. Também fica evidente que as IES públicas então em descompasso, na oferta de cursos de especialização BIM, quando o assunto é a capacitação de profissionas da AECO.

O número de cursos de especialização em BIM, ainda que ofertados em todas as regiões do Brasil, estão bem aquém das necessidades de capacitação profissional exigidas pelo indústria da construção civil, do mesmo modo, a abordagem de muitos cursos pode ser contestada no tocante a alguns pontos, entre eles, a priorização do uso de softwares em detrimento as mudanças do processo de trabalho.

O ensino de BIM, em cursos de pós-graduação lato sensu, não precisa se resumir a utilização de softwares ou de uma determinada plataforma. Muitos cursos não tem explorando a essência do BIM (colaboração, interoperabilidade e parametrização), ao mesmo tempo, pouco se esforçando para integrá-los em uma abordagem universal (OpenBIM). O grande desafio dos cursos de especialização em BIM no Brasil é o de apresentar aos profissionas da AECO um fluxo de trabalho aberto, não importando quais softwares os diversos profissionais estejam trabalhando.

Os problemas relacionados a falta de compreensão do conceito BIM são os obstáculos mais significativos a serem superados (KYMMELL, 2008 apud BARISON, 2015), porque entender a ideia é mais importante do que dominar o uso do software BIM (HIETANEN; DROGEMULLER, 2008 apud BARISON, 2015).

\section{REFERENCIAS}

ADDOR, M. et al. Colocando o “i” no BIM. Revista arq.urb, São Paulo, n. 4, p. 104-115, 2010 .

BARISON, M. Introdução de modelagem da informação da construção (BIM) no currículo. 2015. 390 f. Tese (Doutorado) - Universidade de São Paulo, SP, 2015.

BENEDETTO, H.; BERNARDES, M. M. E. S.; PIRES, R. W. Ensino de BIM no Brasil: Análise do Cenário Acadêmico. Informática na Educação: teoria \& prática, Porto Alegre, v. 20, n. 2, p. 70-84, jan./jul. 2017. 
BRASIL, Conselho Nacional de Educação. Resolução CNE/CES nº 1, de 06 de abril de 2018a. Estabelece diretrizes e normas para a oferta dos cursos de pós-graduação lato sensu denominados cursos de especialização. Brasília: Diário Oficial da União, 06 abr. 2018a. Disponível em: <http://portal.mec.gov.br/docman/abril-2018-pdf/85591-rces001-18/file>. Acesso em: 10 ago. 2020.

Decreto $\mathrm{n}^{\circ}$ 9.377, de 17 de maio de 2018b. Institui a Estratégia Nacional de Disseminação do Building Information Modelling. Diário Oficial da União, Brasília, 18 mai. 2018b. Seção 1, p 3.

E-ZIGURAT, 2019. IFC e interoperabilidade BIM. Disponível em: https://www.ezigurat.com/blog/pt-br/ifc-e-interoperabilidade-bim/. Acesso em: 15 de ago. de 2020.

JAYME, I.; FREIRE, F. BIM no ensino de arquitetura: um estudo sobre a inserção da metodologia em universidades públicas brasileiras. 2019. 10 f. TCC (Especialização) Universidade Tecnológica Federal do Paraná, Curitiba, 2019.

RUSCHEL, R. A especialização Master BIM Especialista implementada no ISITEC. In: ENCONTRO NACIONAL SOBRE O ENSINO DE BIM. 1, 2018, online. Pesquisa...Online, 2018.

SUCCAR, B. Building information modelling framework: A research and delivery foundation for industry stakeholders. Automation in Construction, Amsterdam, v. 18, n. 3, p. 357-375, 2009. 